\title{
Postload Glycated Albumin as an Alternate Measure for Diabetes Screening in a Chinese Population
}

\author{
Hang Su $(\mathbb{D}$, Junling Tang, Xiaojing Ma $\mathbb{D}$, Xingxing He $\mathbb{D}$, Lingwen Ying $\mathbb{D}$, Yufei Wang $(\mathbb{D}$, \\ Yuqian Bao $\mathbb{D}$, and Jian Zhou
}

Department of Endocrinology and Metabolism, Shanghai Clinical Center for Diabetes, Shanghai Diabetes Institute, Shanghai Key Laboratory of Diabetes Mellitus, Shanghai Jiao Tong University Affiliated Sixth People's Hospital, Shanghai 200233, China

Correspondence should be addressed to Xiaojing Ma; maxiaojing@sjtu.edu.cn and Jian Zhou; zhoujian@sjtu.edu.cn

Received 11 December 2017; Accepted 3 May 2018; Published 22 May 2018

Academic Editor: Secundino Cigarran

Copyright @ 2018 Hang Su et al. This is an open access article distributed under the Creative Commons Attribution License, which permits unrestricted use, distribution, and reproduction in any medium, provided the original work is properly cited.

\begin{abstract}
In previous epidemiological screening in China, glycated albumin (GA) was mostly detected during the fasting state. This strict restriction causes some problems with diabetes screening. It is unclear if GA could help improve the efficiency of screening for diabetes for subjects who are not in the fasting state. The present study analyzed the differences between fasting and postload $(30,60,120$, and $180 \mathrm{~min}) \mathrm{GA}$ levels. A total of 691 participants were enrolled in the present study. The Bland-Altman difference plots revealed that $95.4,94.8,93.6$, and $93.9 \%$ of data points were within the limits of agreement for each time point. The receiver operating characteristic curve showed that the areas under the curve (AUC) for baseline GA and postload GA for every time point were 0.822 (95\% CI 0.791-0.849), 0.821 (95\% CI 0.790-0.848), 0.833 (95\% CI 0.803-0.860), 0.840 (95\% CI $0.811-0.867)$, and 0.840 (95\% CI $0.810-0.867)$, with sensitivities of $67.5,68.1,69.3,71.6$, and $69.3 \%$, respectively. There was no difference between the baseline and postload GA levels in either AUC or sensitivity (all $p>0.05$ ). In conclusion, postload serum GA levels were in good agreement with those at baseline, and thus, it may be reasonable to employ nonfasting measurements of GA levels for diabetes screening.
\end{abstract}

\section{Introduction}

For clinical monitoring indicators, the value of a nonfasting measurement is an important determinant of its extended application in the clinical setting. For example, in recent years, nonfasting lipid profile measurements have been vigorously promoted by numerous studies [1]. Similarly, the levels of glycated hemoglobin $\mathrm{A}_{1 \mathrm{c}}\left(\mathrm{HbA}_{1 \mathrm{c}}\right)$, a standard blood glucose monitoring indicator, have been shown to remain stable after a glucose load in a previous study [2]. It is expected that increased flexibility regarding blood sampling time could improve patient adherence.

As an emerging indicator, glycated albumin (GA) has been proposed to be an effective supplement to $\mathrm{HbA}_{1 \mathrm{c}}$ for blood glucose monitoring. Recently, many studies have suggested that GA may be an effective marker for screening for diabetes mellitus (DM) [3-5]. We previously reported that GA was a sensitive and specific indicator for diabetes screening in Chinese subjects [5]. In previous epidemiological screening in China, GA was mostly detected during the fasting state. This strict restriction causes some problems with diabetes screening. It is unclear if GA could help improve the efficiency of screening for diabetes for subjects who are not in the fasting state.

However, few studies have explored the changes in serum GA levels after a glucose load, especially in the Chinese population. Nevertheless, we have no information on the value of postload GA for diabetes screening and diagnosis. Published data include only small sample-sized studies that have used relatively simple evaluations and drawn inconsistent conclusions. Some studies found no significant changes in serum GA levels after a glucose load [6,7], whereas Hashimoto et al. [8] showed that the 2-hour postprandial GA levels were slightly increased in diabetes patients with poor glycemic control. Therefore, to provide evidence for clinical application of GA measurements, this study aimed at comparing 
results between fasting and nonfasting GA levels and providing an analysis of diabetes screening efficiency for postload GA in a large population sample with different glucose tolerance statuses.

\section{Materials and Methods}

2.1. Study Population. The study population comprised of 691 participants, including 178 with normal glucose tolerance, 178 with impaired glucose regulation, and 335 who were newly diagnosed with antihyperglycemic agent-naive DM, who presented in the clinic of the Department of Endocrinology and Metabolism of Shanghai Jiao Tong University Affiliated Sixth People's Hospital from January 2014 to January 2016. The population was restricted to those without a history of thyroid dysfunction, chronic liver disease, nephrotic syndrome, hypoalbuminemia, tumors, mental disorders, acute infection, pregnancy, or glucocorticoids therapy.

This study was approved by the Ethics Committee of Shanghai Jiao Tong University Affiliated Sixth People's Hospital. Written informed consents were provided by all participants prior to enrollment.

2.2. Physical and Laboratory Assessments. All participants completed a standard questionnaire in the outpatient department after overnight ( $8-10$ hours) fasting. The questionnaire collected details of the patients' medical histories, including the patients' histories of past and present illnesses, medication histories, and family histories. Physical examination included measurements of height, weight, and blood pressure. The body mass index (BMI) was calculated as weight/ height ${ }^{2}\left(\mathrm{~kg} / \mathrm{m}^{2}\right)$.

Blood samples were collected to measure the fasting levels of plasma glucose $\left(\mathrm{PG}_{0}\right)$, serum $\mathrm{GA}\left(\mathrm{GA}_{0}\right)$, and $\mathrm{HbA}_{1 \mathrm{c}}$. Postload levels of plasma glucose $\left(\mathrm{PG}_{30}, \mathrm{PG}_{60}, \mathrm{PG}_{120}\right.$, and $\left.\mathrm{PG}_{180}\right)$ and serum GA $\left(\mathrm{GA}_{30}, \mathrm{GA}_{60}, \mathrm{GA}_{120}\right.$, and $\left.\mathrm{GA}_{180}\right)$ were measured at 30,60,120, and $180 \mathrm{~min}$ after administration of $75 \mathrm{~g}$ of oral glucose (oral glucose tolerance test, OGTT). The plasma glucose levels were immediately obtained via the glucose oxidase method (Kehua Biological Engineering Co., Ltd., Shanghai, China) using the Glamour 2000 biochemical autoanalyzer. GA levels were measured via an enzymatic method using an enzyme-based assay kit (Lucica GA-L, Asahi Kasei Pharma, Tokyo, Japan) on a 7600-120 autoanalyzer (Hitachi, Tokyo, Japan) with intra- and interassay coefficients of variation (CVs) of $1.47-3.30 \%$ and $1.95-4.73 \%$, respectively. $\mathrm{HbA}_{1 \mathrm{c}}$ levels were detected by high-performance liquid chromatography (HPLC, Variant II hemoglobin analyzer; Bio-Rad, Hercules, CA, USA) with intra- and interassay CVs of $0.55-2.58 \%$ and $0.75-$ $3.39 \%$, respectively.

2.3. Diagnostic Criteria. DM and impaired glucose regulation were diagnosed according to the 1999 World Health Organization criteria [9].

2.4. Consistency Analysis. A mountain plot was created by computing a percentile rank for ranked differences between paired postload and baseline GA levels and cumulative percentages ( $y$-axis values) against the ranked differences ( $x$-axis values) to evaluate the agreement between postload and baseline GA levels [10]. The Bland-Altman difference plot was used to depict the differences between the paired postload and baseline GA levels after $\log$ transformation (baseline $\log G A$ minus postload $\log \mathrm{GA}$ result along the $y$-axis against the average of the baseline $\log \mathrm{GA}$ and postload $\log \mathrm{GA}$ along the $x$-axis) [11]. The $95 \%$ confidence intervals (CIs) for the difference ranges (the sample mean difference \pm 1.96 standard deviation) reflected the $95 \%$ probability range in which the mean difference population parameter lies [12]. If more than $95 \%$ of data points fell within these limits of agreement, there was not a significant systematic difference between the two points of time for the measurement.

2.5. Statistical Analysis. All statistical analyses were performed using SPSS 19.0 and MedCalc statistical software version 15.2. Data are presented as the means \pm standard deviation. Each variable was examined for a normal distribution, and pair analyses were carried out using a paired Student's $t$-test and Wilcoxon signed-rank sum test. The chi-square test was used for intergroup comparisons of categorical variables. Intergroup comparisons of skewed data were made using the Kruskal-Wallis test. The absolute relative errors (AREs) were calculated to assess the differences in the postload and baseline GA levels. Spearman correlation analysis was performed to explore the agreement in the postload GA levels. The receiver operating characteristic (ROC) curve was plotted to assess the power of the postload GA as a screening test to discriminate diabetes patients from nondiabetes patients. The mountain plot and Bland-Altman difference plots were used to identify the bias in the postload GA levels. A two-tailed $p$ value of $<0.05$ was considered to be statistically significant.

\section{Results}

3.1. Clinical Characteristics of the Study Participants. The study included a total of 691 participants of 323 men and 368 women, including 356 without DM (non-DM group) and 335 with DM. Compared to the non-DM participants, patients in the DM group were older and had significantly higher BMI, systolic blood pressure, diastolic blood pressure, and $\mathrm{HbA}_{1 \mathrm{c}}$, and PG and GA levels at all measurement time points (all $p<0.001$, Table 1 ).

3.2. Analysis of Agreement for the Postload and Baseline GA Levels. The obtained measurements demonstrated that GA levels at 30 and $60 \mathrm{~min}$ postload were slightly elevated compared with the baseline levels in both the non-DM and DM groups (all $p<0.01$, Figure 1). Spearman correlation analysis revealed that baseline GA levels were positively associated with postload GA levels at every time point for all participants $(r=0.977$ to 0.981 , all $p<0.01)$. The AREs for postload GA levels at $30,60,120$, and $180 \mathrm{~min}$ were $2.6 \pm 2.1 \%, 2.8 \pm$ $2.2 \%, 2.8 \pm 2.2 \%$, and $2.6 \pm 2.3 \%$, respectively. The mountain plot showed that the mountain peaked at approximately $x=0$ and was symmetric around the line of $x=0$ without significant shifts. Most of the differences were within \pm 1 
TABle 1: Demographic and clinical characteristics of study participants.

\begin{tabular}{|c|c|c|c|c|}
\hline Variable & Total $(n=691)$ & Non-DM $(n=356)$ & $\mathrm{DM}(n=335)$ & $p$ value \\
\hline$n$ (men/women) & $323 / 368$ & $141 / 215$ & $182 / 153$ & $<0.001$ \\
\hline Age (years) & $50.5 \pm 13.3$ & $46.7 \pm 14.0$ & $53.6 \pm 11.7$ & $<0.001$ \\
\hline BMI $\left(\mathrm{kg} / \mathrm{m}^{2}\right)$ & $24.7 \pm 3.3$ & $24.2 \pm 3.3$ & $25.3 \pm 3.2$ & $<0.001$ \\
\hline Systolic blood pressure (mmHg) & $130.1 \pm 17.9$ & $127.1 \pm 17.3$ & $135.3 \pm 17.6$ & $<0.001$ \\
\hline Diastolic blood pressure (mmHg) & $79.4 \pm 10.8$ & $77.0 \pm 10.5$ & $82.0 \pm 10.6$ & $<0.001$ \\
\hline $\mathrm{HbA}_{1 \mathrm{c}}(\%)$ & $6.1 \pm 0.9$ & $5.6 \pm 0.4$ & $6.7 \pm 1.0$ & $<0.001$ \\
\hline $\mathrm{HbA}_{1 \mathrm{c}}(\mathrm{mmol} / \mathrm{mol})$ & $43 \pm 10$ & $38 \pm 5$ & $49 \pm 11$ & $<0.001$ \\
\hline $\mathrm{PG}_{0}(\mathrm{mmol} / \mathrm{L})$ & $6.6 \pm 1.5$ & $5.7 \pm 0.6$ & $7.7 \pm 1.5$ & $<0.001$ \\
\hline $\mathrm{PG}_{30}(\mathrm{mmol} / \mathrm{L})$ & $11.2 \pm 2.6$ & $9.7 \pm 1.7$ & $12.8 \pm 2.3$ & $<0.001$ \\
\hline $\mathrm{PG}_{60}(\mathrm{mmol} / \mathrm{L})$ & $12.9 \pm 4.1$ & $10.1 \pm 2.7$ & $15.9 \pm 3.0$ & $<0.001$ \\
\hline $\mathrm{PG}_{120}(\mathrm{mmol} / \mathrm{L})$ & $11.0 \pm 4.8$ & $7.4 \pm 1.8$ & $14.9 \pm 3.9$ & $<0.001$ \\
\hline $\mathrm{PG}_{180}(\mathrm{mmol} / \mathrm{L})$ & $7.5 \pm 3.8$ & $5.2 \pm 1.6$ & $9.9 \pm 3.9$ & $<0.001$ \\
\hline $\mathrm{GA}_{0}(\%)$ & $16.2 \pm 3.1$ & $14.6 \pm 1.9$ & $17.8 \pm 3.3$ & $<0.001$ \\
\hline $\mathrm{GA}_{30}(\%)$ & $16.3 \pm 3.2$ & $14.7 \pm 2.0$ & $18.0 \pm 3.3$ & $<0.001$ \\
\hline $\mathrm{GA}_{60}(\%)$ & $16.3 \pm 3.2$ & $14.7 \pm 1.9$ & $18.1 \pm 3.3$ & $<0.001$ \\
\hline $\mathrm{GA}_{120}(\%)$ & $16.3 \pm 3.2$ & $14.6 \pm 1.8$ & $18.1 \pm 3.4$ & $<0.001$ \\
\hline $\mathrm{GA}_{180}(\%)$ & $16.1 \pm 3.1$ & $14.4 \pm 1.8$ & $17.8 \pm 3.3$ & $<0.001$ \\
\hline
\end{tabular}

Data are presented as mean \pm standard. BMI: body mass index; $\mathrm{HbA}_{1 c}$ : glycated hemoglobin $\mathrm{A}_{1 \mathrm{c}}$; $\mathrm{PG}_{0}$ : fasting plasma glucose; $\mathrm{PG}_{30}$ : 30 min postload plasma glucose; $\mathrm{PG}_{60}: 60 \mathrm{~min}$ postload plasma glucose; $\mathrm{PG}_{120}: 120 \mathrm{~min}$ postload plasma glucose; $\mathrm{PG}_{180}: 180 \mathrm{~min}$ postload plasma glucose; $\mathrm{GA}_{0}$ : fasting glycated albumin; $\mathrm{GA}_{30}: 30$ min glycated albumin; $\mathrm{GA}_{60}: 60$ min glycated albumin; $\mathrm{GA}_{120}: 120$ min glycated albumin; $\mathrm{GA}_{180}: 180$ min glycated albumin.

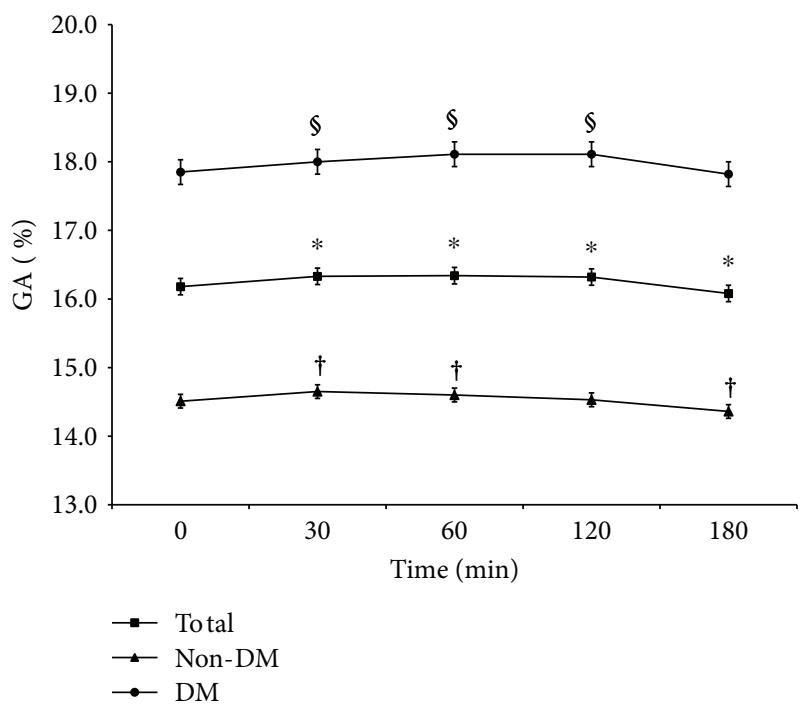

FIGURE 1: Serum GA levels for the entire study population, the DM group, and the non-DM group. Data are presented as the means \pm standard deviation. ${ }^{*} p<0.01$ versus fasting GA levels in the entire study population; ${ }^{\dagger} p<0.01$ versus fasting GA levels in the non$\mathrm{DM}$ group; ${ }^{\circledR} p<0.01$ versus fasting GA levels in the DM group.

standard deviation, indicating high agreement between the postload and baseline GA levels (Figure 2). The BlandAltman difference plots revealed that the mean differences and 95\% CIs between the postload GA levels at every time point and baseline GA measurements after log transformation were $0.004 \%(-0.023-0.031 \%), 0.004 \%(-0.025-$ $0.033 \%), 0.003 \%(-0.026-0.033 \%)$, and $-0.003 \%(-0.032-$ $0.027 \%)$, respectively. On this graph, 95.4, 94.8, 93.6, and

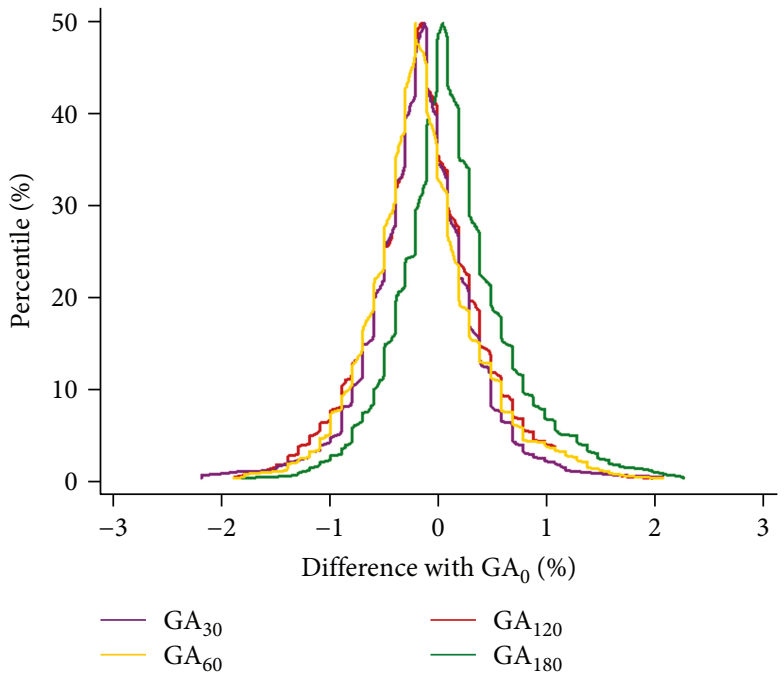

FIgURE 2: Mountain plot of agreement between postload and baseline GA levels in all participants (cumulative percentages with a lower or equal value to the score under consideration along the $y$-axis against the postload GA levels minus baseline GA levels along the $x$-axis).

$93.9 \%$ of the data points for $\mathrm{GA}_{30}, \mathrm{GA}_{60}, \mathrm{GA}_{120}$, and $\mathrm{GA}_{180}$ fell within the limits of agreement, respectively (Figure 3 ).

3.3. Screening of Efficiency of Postload and Baseline GA for Diabetes. The ROC curve was plotted to examine the predictive values of the postload and baseline GA levels for identifying undiagnosed diabetes. The optimal cut-off point of the baseline GA was $16.3 \%$ with a sensitivity, specificity, positive predictive value, and negative predictive value of $67.5 \%$ 

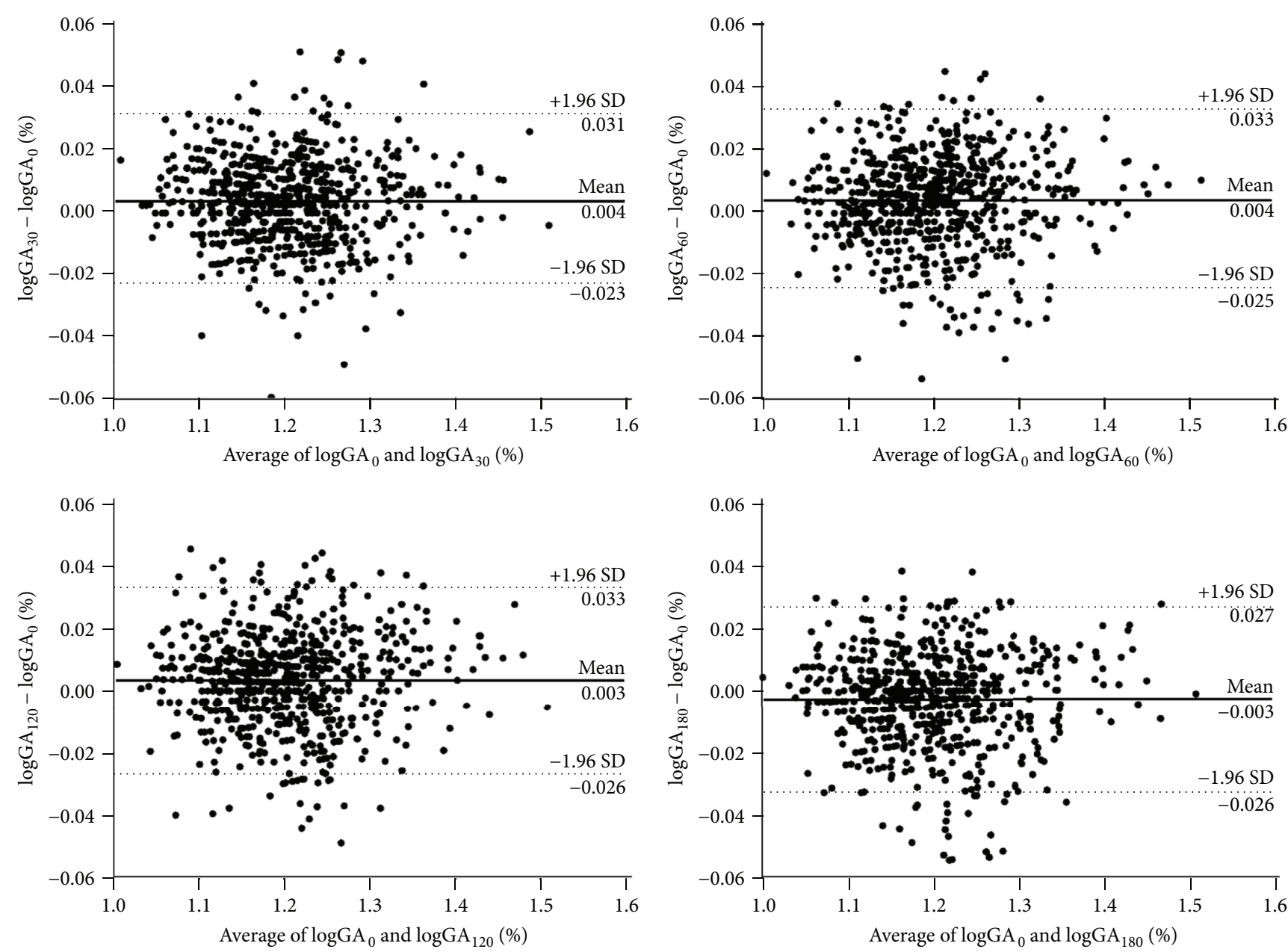

FIGURE 3: Bland-Altman difference plots showing the differences between baseline and postload GA levels after log transformation for all participants (baseline $\log \mathrm{GA}$ minus postload $\log \mathrm{GA}$ result along the $y$-axis against the average of the baseline logGA and postload logGA along the $x$-axis). Horizontal lines are drawn at the mean difference (solid line) and the limits of agreement (both upper and lower, dashed lines).

TABLE 2: Diagnostic indices for screening diabetes with postload and baseline GA.

\begin{tabular}{lcccc}
\hline Criteria & Sensitivity (\%) & Specificity (\%) & Positive predictive value (\%) & Negative predictive value (\%) \\
\hline $\mathrm{GA}_{0} \geq 16.3 \%$ & 67.5 (95\% CI: 62.2-72.5\%) & $83.4 \%$ (95\% CI: 79.2-87.1\%) & 79.3 (95\% CI: 74.1-83.9\%) & $73.2 \%(95 \%$ CI: 68.6-77.4\%) \\
$\mathrm{GA}_{30} \geq 16.4 \%$ & 68.1 (95\% CI: 62.8-73.0\%) & $81.5 \%$ (95\% CI: 77.0-85.4\%) & 77.6 (95\% CI: 72.4-82.2\%) & $73.1 \%(95 \% \mathrm{CI}: 68.4-77.4 \%)$ \\
$\mathrm{GA}_{60} \geq 16.4 \%$ & 69.3 (95\% CI: 64.0-74.2\%) & $80.6 \%$ (95\% CI: 76.1-84.6\%) & 77.1 (95\% CI: 71.9-81.7\%) & $73.6 \%(95 \% \mathrm{CI}: 68.9-77.9 \%)$ \\
$\mathrm{GA}_{120} \geq 16.3 \%$ & 71.6 (95\% CI: 66.5-76.4\%) & $83.4 \%$ (95\% CI: 79.2-87.1\%) & 80.3 (95\% CI: 75.3-84.6\%) & $75.8 \%(95 \% \mathrm{CI}: 71.2-79.9 \%)$ \\
$\mathrm{GA}_{180} \geq 16.2 \%$ & 69.3 (95\% CI: 64.0-74.2\%) & $86.0 \%$ (95\% CI: 82.2-89.6\%) & 82.6 (95\% CI: 77.6-86.8\%) & $74.9 \%(95 \% \mathrm{CI}: 70.4-79.0 \%)$ \\
\hline
\end{tabular}

GA: glycated albumin; CI: confidence interval.

(95\% CI: 62.2-72.5\%), 83.4\% (95\% CI: 79.2-87.1\%), $79.3 \%$ (95\% CI: $74.1-83.9 \%$ ), and $73.2 \%$ (95\% CI: $68.6-77.4 \%$ ), respectively. The diagnostic indices for screening diabetes with postload GA are shown in Table 2. The McNemar test revealed that the postload GA levels at every time point exhibited an equivalent sensitivity as baseline GA for identifying diabetes (all $p>0.05$ ). The areas under the curve (AUC) for baseline GA and postload GA at every time point were 0.822 (95\% CI 0.791-0.849), 0.821 (95\% CI $0.790-$ 0.848 ), 0.833 (95\% CI $0.803-0.860), 0.840$ (95\% CI $0.811-$
0.867), and 0.840 (95\% CI $0.810-0.867$ ), respectively. There were no differences in the AUC for the baseline GA levels and the postload GA levels at every time point $(p=0.965$, $0.620,0.413$, and 0.413 , Figure 4 ).

\section{Discussion}

The current study provides the first evaluation of the screening efficiency of serum GA levels after glucose load in a Chinese population with differing glucose tolerance 


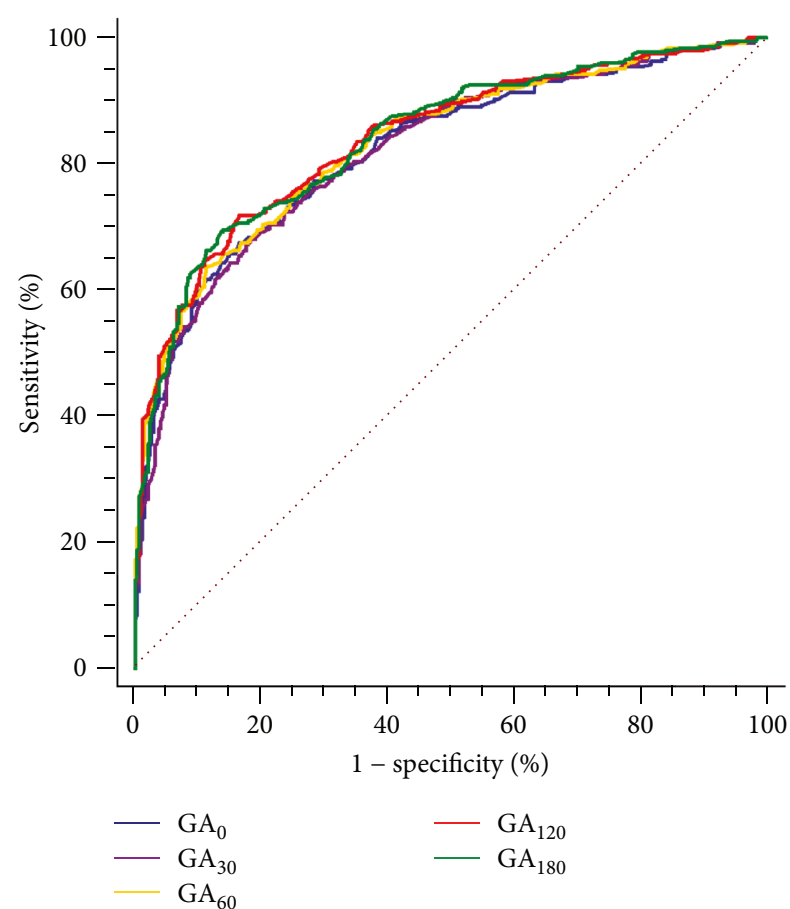

FIGURE 4: ROC curves of postload and baseline GA for screening diabetes.

statuses. The results demonstrated that the AREs for postload GA levels at all measurement time points $(30,60,120$, and 180 min postload) were all within $5 \%$, and more than $93.6 \%$ of data points from each measurement time point were found to be within the limits of agreement of the baseline level. There was no difference between the sensitivity of the baseline versus postload GA for screening diabetes. Moreover, the ROC curve showed equal levels of baseline and postload GA for screening diabetes, which indicates that postload GA can also guide diabetes screening.

Serum GA can reflect short-term (2-3 weeks) mean glycemic levels [13-18], and the measurement of GA levels has distinctive advantages in patients with newly diagnosed DM, marked glycemic excursion, treatment adjustment, and stress [19]. Recent studies have focused on the role of GA as a direct pathological harmful factor in the development of vascular complications in DM $[14,20]$. Additionally, GA cannot only predict the development but also indicate the severity of DM complications [21-24]. Recently, many studies have assessed the value of GA as an effective marker for DM screening $[3-5,25]$. Our recently study also showed that the additional measurement of GA could help prevent the misdiagnosis of diabetes [26], which is consistent with the findings of the present study. The different population constituents may explain the cut-off inconsistency. In addition, we used the ROC curve for further verification. The result suggested that nonfasting GA could also help indirect diabetes screening, which improves the efficiency and convenience of assessing diabetes.

To date, few studies have assessed the agreement between postload and baseline GA levels. Shima et al. [6] investigated the diurnal variation in GA levels in $15 \mathrm{DM}$ patients via
HPLC analysis and reported almost no change in the GA levels. A study in Taiwan involving 12 individuals without DM who underwent $75 \mathrm{~g}$ of OGTT showed via pair analysis that the GA levels were similar in fasting and postprandial samples [7]. However, Hashimoto et al. [8] found that the GA levels increased between preprandial and $2 \mathrm{~h}$ postprandial specimens in 16 Japanese DM patients with poor glycemic control $(p<0.05)$, especially after breakfast $(p<0.05)$ and observed a significant correlation between the variation ranges of GA and blood glucose measurements $(r=0.322$, $p=0.021)$. The present study utilized the Wilcoxon signedrank sum test and Spearman correlation to evaluate the central tendency and correlation, respectively. The discrete tendency was evaluated by generating a mountain plot and Bland-Altman difference plots. We confirmed that although there was a slight increase, postload GA levels were in good agreement with baseline GA levels. We further investigated the screening efficiency of postload GA in our study population of 691 Chinese individuals with varying glucose metabolism statuses. The results indicated that it is reasonable to employ nonfasting measurements of GA levels for diabetes screening.

These inconsistencies in the variation of GA after glucose load may be attributed to different factors, such as variations in blood sampling, storage processes, and methodological assessment. The mechanisms responsible for the minor changes in GA levels after application of a glucose load remain unclear and may be related to different glycated reactions of $\mathrm{GA}$ and $\mathrm{HbA}_{1 \mathrm{c}}$. In $\mathrm{HbA}_{1 \mathrm{c}}$, valine is glycated, whereas lysine is glycated in GA. This difference in the glycated amino acid may lead to the different kinetics of the early Amadori reaction. Day et al. [27] reported that the glycation reaction of GA progressed approximately 10 times faster than that of $\mathrm{HbA}_{1 \mathrm{c}}$ in a SpragueDawley rat model of DM. This is believed to be because when the elevated blood glucose level decreases, an unstable product of early $\mathrm{HbA}_{1 \mathrm{c}}$ through the Amadori reaction is reversibly dissociated to hemoglobin and glucose [28], whereas unstable GA produced from albumin and glucose rapidly goes through the irreversible reaction, and stable GA is produced [14]. Further studies are needed to fully elucidate the underlying mechanisms.

There are some limitations in this study. First, this was a single-center study, and data from multiple centers are needed to further confirm the findings. Second, serum GA levels were evaluated at only four time points after the glucose load. Data from more measurement time points could provide a more complete picture of the GA variation after the glucose load.

\section{Conclusion}

This study showed that postload GA levels were in good agreement with baseline GA levels in Chinese individuals with different glucose tolerance statuses. These results support that the nonfasting GA measurement has improved convenience and equal effectiveness for diabetes assessments. Thus, the nonfasting GA measurement may contribute to greater patient adherence to diabetes screening. Finally, this 
study provides a foundation for further application of GA measurements for diabetes screening.

\author{
Abbreviations \\ ARE: Absolute relative error \\ AUC: Area under the curve \\ BMI: Body mass index \\ CI: $\quad$ Confidence interval \\ CV: Coefficients of variation \\ DM: Diabetes mellitus \\ GA: $\quad$ Glycated albumin \\ $\mathrm{HbA}_{1 \mathrm{c}}$ : Glycated hemoglobin $\mathrm{A}_{1 \mathrm{c}}$ \\ HPLC: High-performance liquid chromatography \\ OGTT: Oral glucose tolerance test \\ PG: $\quad$ Plasma glucose \\ ROC: Receiver operating characteristic.
}

\section{Ethical Approval}

All procedures performed in studies involving human participants were in accordance with the ethical standards of the Ethics Committee of Shanghai Jiao Tong University Affiliated Sixth People's Hospital and in accordance with the 1964 Helsinki declaration and its later amendments or comparable ethical standards.

\section{Consent}

Informed consents were obtained from all individual participants in the study.

\section{Disclosure}

The authors have no employment or leadership, consultant or advisory role, stock ownership, honoraria, expert testimony, and patents to declare. The funding organizations played no role in the study design; the collection, analysis, or interpretation of data; the writing of the manuscript; or the decision to submit the manuscript for publication.

\section{Conflicts of Interest}

The authors declare that they have no conflicts of interest.

\section{Authors' Contributions}

Jian Zhou and Xiaojing Ma designed the study. Junling Tang, Hang Su, Lingwen Ying, and Xingxing He collected the data. Hang Su analyzed the data and wrote the draft. Xiaojing Ma, Junling Tang, and Yufei Wang provided technical support. Jian Zhou and Yuqian Bao revised the paper and contributed to the discussion. All of the authors read and approved the final manuscript. Hang Su and Junling Tang contributed equally to this work.

\section{Acknowledgments}

This work was funded by the Shanghai Municipal Commission of Health and Family Planning General Program
(201540093) and the Shanghai Municipal Education Commission-Gaofeng Clinical Medicine Grant support (20161430).

\section{References}

[1] B. G. Nordestgaard, A. Langsted, S. Mora et al., "Fasting is not routinely required for determination of a lipid profile: clinical and laboratory implications including flagging at desirable concentration cut-points-a joint consensus statement from the European Atherosclerosis Society and European Federation of Clinical Chemistry and Laboratory Medicine," European Heart Journal, vol. 37, no. 25, pp. 1944-1958, 2016.

[2] I. N. Scobie, F. Onyanga-Omara, M. Singaraveloo, A. R. Forrest, A. C. MacCuish, and W. G. Manderson, "Changes in glycosylated haemoglobin after oral glucose load," British Medical Journal, vol. 283, no. 6296, pp. 877-878, 1981.

[3] N. Mukai, M. Yasuda, T. Ninomiya et al., "Thresholds of various glycemic measures for diagnosing diabetes based on prevalence of retinopathy in community-dwelling Japanese subjects: the Hisayama Study," Cardiovascular Diabetology, vol. 13, no. 1, p. 45, 2014.

[4] H. Ikezaki, N. Furusyo, T. Ihara et al., "Glycated albumin as a diagnostic tool for diabetes in a general Japanese population," Metabolism, vol. 64, no. 6, pp. 698-705, 2015.

[5] X. J. Ma, J. M. Pan, Y. Q. Bao et al., “Combined assessment of glycated albumin and fasting plasma glucose improves the detection of diabetes in Chinese subjects," Clinical and Experimental Pharmacology and Physiology, vol. 37, no. 10, pp. 974979, 2010.

[6] K. Shima, N. Ito, F. Abe et al., "High-performance liquid chromatographic assay of serum glycated albumin," Diabetologia, vol. 31, no. 8, pp. 627-631, 1988.

[7] W. C. Wu, W. Y. Ma, J. N. Wei et al., "Serum glycated albumin to guide the diagnosis of diabetes mellitus," PLoS One, vol. 11, no. 1, article e0146780, 2016.

[8] K. Hashimoto, K. Tanikawa, J. Nishikawa, Y. Chen, T. Suzuki, and M. Koga, "Association of variation range in glycated albumin (GA) with increase but not decrease in plasma glucose: implication for the mechanism by which GA reflects glycemic excursion," Clinical Biochemistry, vol. 48, no. 6, pp. 397-400, 2015.

[9] World Health Organization and Department of Noncommunicable Disease Surveillance, "Definition, diagnosis and classification of diabetes mellitus and its complication: report of a WHO Consultation," in Part1: Diagnosis and Classification of Diabetes Mellitus, p. 59, World Health Organization, Geneva, 1999.

[10] S. M. Haley, P. Ni, W. J. Coster, R. Black-Schaffer, H. Siebens, and W. Tao, "Agreement in functional assessment: graphic approaches to displaying respondent effects," American Journal of Physical Medicine \& Rehabilitation, vol. 85, no. 9, pp. 747-755, 2006.

[11] J. Martin Bland and D. G. Altman, "Statistical methods for assessing agreement between two methods of clinical measurement," The Lancet, vol. 327, no. 8476, pp. 307-310, 1986.

[12] J. M. Bland and D. G. Altman, "Measuring agreement in method comparison studies," Statistical Methods in Medical Research, vol. 8, no. 2, pp. 135-160, 1999.

[13] M. Koga, "1,5-Anhydroglucitol and glycated albumin in glycemia," Advances in Clinical Chemistry, vol. 64, pp. 269301, 2014. 
[14] H. V. Roohk, A. R. Zaidi, and D. Patel, "Glycated albumin (GA) and inflammation: role of GA as a potential marker of inflammation," Inflammation Research, vol. 67, no. 1, pp. 21-30, 2018.

[15] H. Kobayashi, M. Abe, Y. Yoshida, H. Suzuki, N. Maruyama, and K. Okada, "Glycated albumin versus glycated hemoglobin as a glycemic indicator in diabetic patients on peritoneal dialysis," International Journal of Molecular Sciences, vol. 17, no. $5,2016$.

[16] E. Dozio, N. Di Gaetano, P. Findeisen, and M. M. Corsi Romanelli, "Glycated albumin: from biochemistry and laboratory medicine to clinical practice," Endocrine, vol. 55, no. 3, pp. 682-690, 2017.

[17] Y. Mo, X. Ma, H. Li et al., "Relationship between glycated albumin and glycated hemoglobin according to glucose tolerance status: a multicenter study," Diabetes Research and Clinical Practice, vol. 115, pp. 17-23, 2016.

[18] N. Duan, S. N. Zhu, H. X. Li, L. L. Jiao, H. Y. Yang, and Q. Guo, "Assessment of glycated albumin as a useful indicator for renal dysfunction in diabetic and nondiabetic population," Clinical Laboratory, vol. 63, no. 7, pp. 1129-1137, 2017.

[19] J. Zhou, H. Li, X. Zhang et al., "Nateglinide and acarbose are comparably effective reducers of postprandial glycemic excursions in Chinese antihyperglycemic agent-naive subjects with type 2 diabetes," Diabetes Technology \& Therapeutics, vol. 15, no. 6, pp. 481-488, 2013.

[20] S. Chen, M. P. Cohen, and F. N. Ziyadeh, "Amadori-glycated albumin in diabetic nephropathy: pathophysiologic connections," Kidney International, vol. 58, pp. S40-S44, 2000.

[21] H.-j. Yoon, Y.-h. Lee, S. R. Kim et al., "Glycated albumin and the risk of micro- and macrovascular complications in subjects with type 1 diabetes," Cardiovascular Diabetology, vol. 14, no. 1, p. 53, 2015.

[22] I. Shimizu, Y. Hiramatsu, Y. Omori, M. Nakabayashi, and the JGA (Japan Glycated Albumin) Study Group, "Comparison of HbAlc and glycated albumin as a control marker for newborn complications in diabetic women in a multicentre study in Japan (Japan glycated albumin study group: study 2)," Annals of Clinical Biochemistry, 2018.

[23] J. E. Jun, S. E. Lee, Y. B. Lee et al., "Glycated albumin and its variability as an indicator of cardiovascular autonomic neuropathy development in type 2 diabetic patients," Cardiovascular Diabetology, vol. 16, no. 1, p. 127, 2017.

[24] X. Ma, X. Hu, J. Zhou et al., "Glycated albumin is more closely correlated with coronary artery disease than 1,5-anhydroglucitol and glycated hemoglobin A1c," Cardiovascular Diabetology, vol. 14, no. 1, p. 16, 2015.

[25] P. A. C. Freitas, L. R. Ehlert, and J. L. Camargo, "Glycated albumin: a potential biomarker in diabetes," Archives of Endocrinology and Metabolism, vol. 61, no. 3, pp. 296-304, 2017.

[26] X. He, L. Ying, X. Ma et al., “An additional measurement of glycated albumin can help prevent missed diagnosis of diabetes in Chinese population," Clinica Chimica Acta, vol. 475, pp. 188-192, 2017.

[27] J. F. Day, C. G. Ingebretsen, W. R. Ingebretsen, J. W. Baynes, and S. R. Thorpe, "Nonenzymatic glucosylation of serum proteins and hemoglobin: response to changes in blood glucose levels in diabetic rats," Diabetes, vol. 29, no. 7, pp. 524-527, 1980.

[28] K. F. McFarland, E. W. Catalano, J. F. Day, S. R. Thorpe, and J. W. Baynes, "Nonenzymatic glucosylation of serum proteins in diabetes mellitus," Diabetes, vol. 28, no. 11, pp. 1011-1014, 1979. 


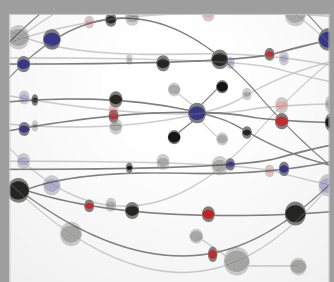

The Scientific World Journal
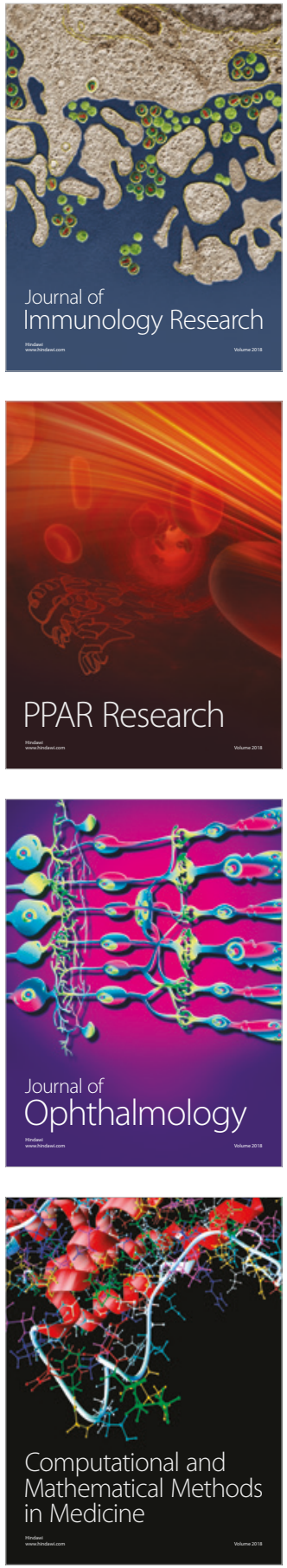

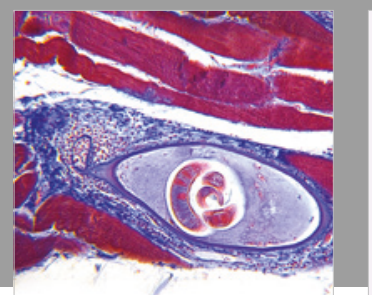

Gastroenterology Research and Practice

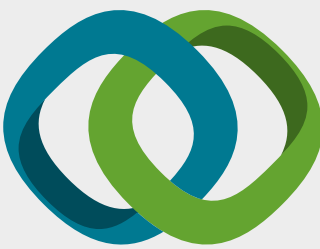

\section{Hindawi}

Submit your manuscripts at

www.hindawi.com
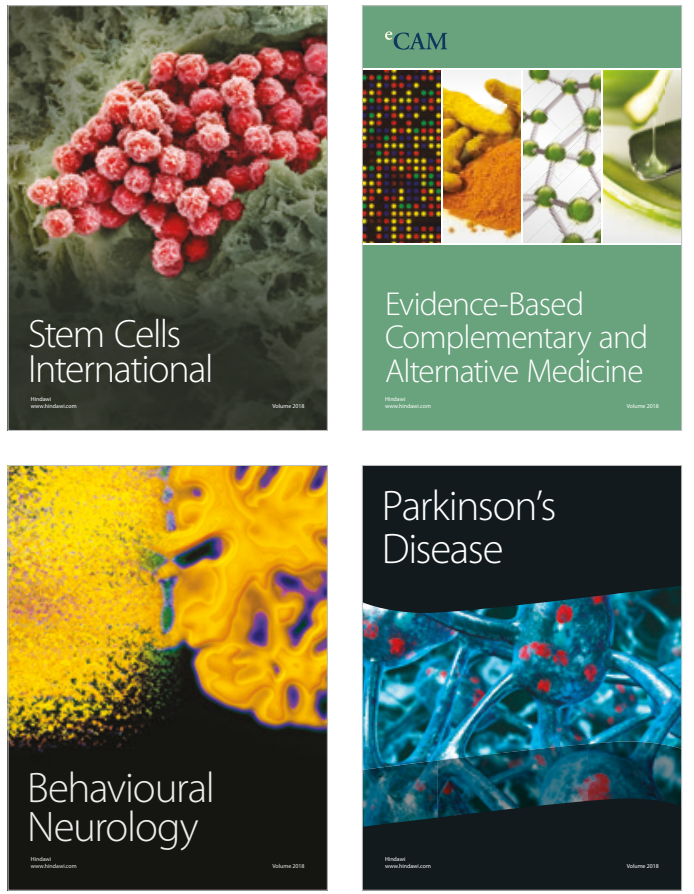

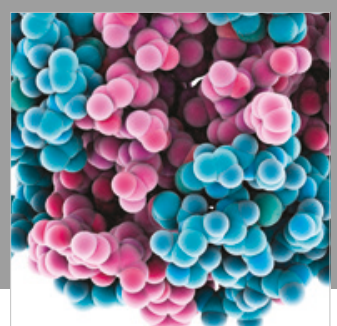

ournal of

Diabetes Research

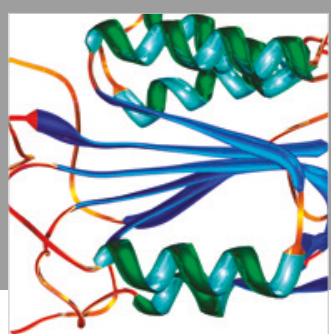

Disease Markers
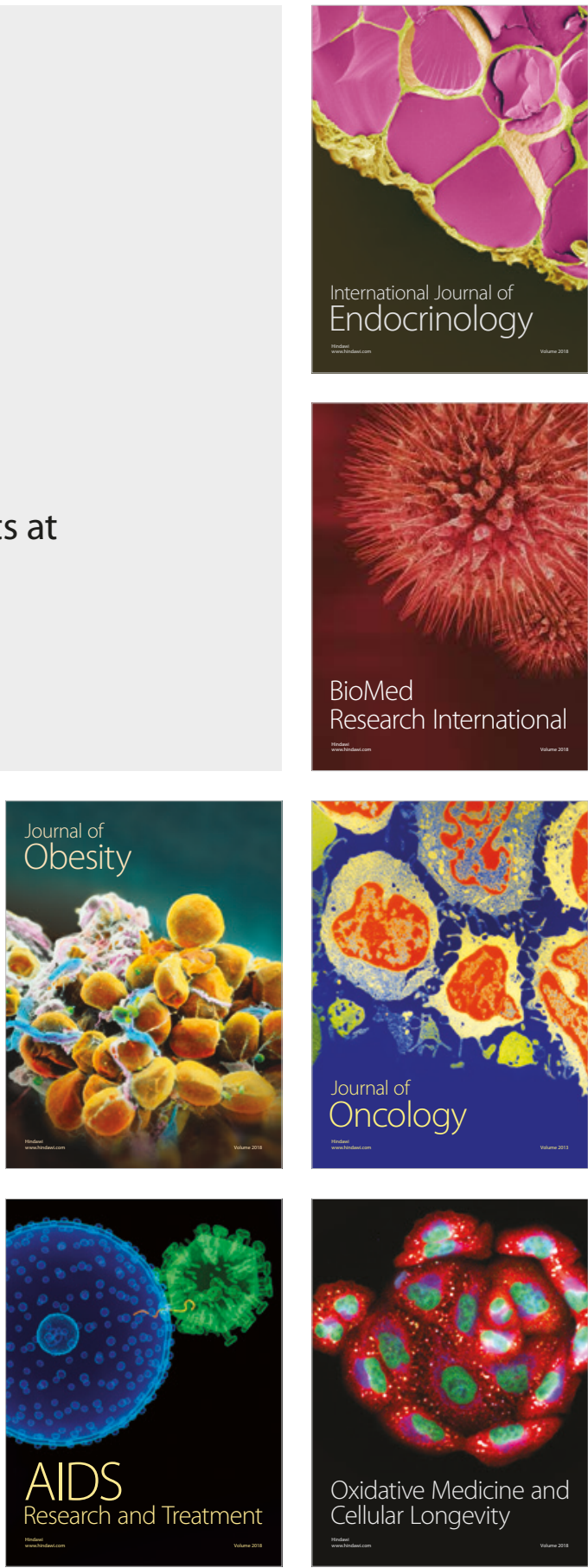